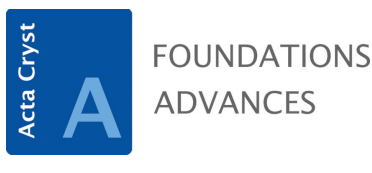

ISSN 2053-2733

Keywords: book review; electrons in solids.

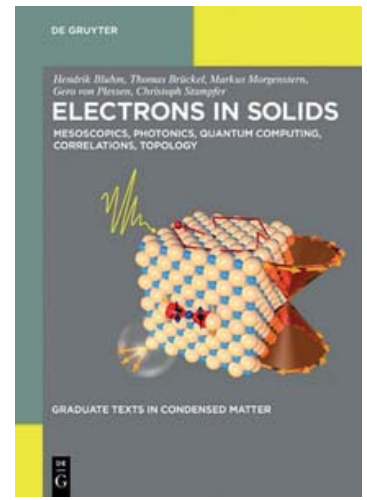

(C) 2020 International Union of Crystallography

\section{Electrons in Solids: Mesoscopics, Photonics, Quantum Computing, Correlations, Topology (Graduate Texts in Condensed Matter). By Hendrik Bluhm, Thomas Brückel, Markus Morgenstern, Gero Plessen and Christoph Stampfer. De Gruyter, 2019. Paperback, pp. 393. Price EUR 59.65. ISBN 978-3-11-043831-4.}

\author{
Piero Macchi*
}

Department of Chemistry, Materials and Chemical Engineering, Politecnico di Milano, Via Mancinelli 7, Milano, 20131, Italy. ${ }^{*}$ Correspondence e-mail: piero.macchi@polimi.it

There are many books sharing the same or a similar title, but their content and focus may vary substantially. Therefore, one should carefully read an abstract or the list of topics before approaching this book, as well as the other books with the same title, of course. Each of them sheds light on different aspects of the fascinating world that rules the physics of the solid state and material science. In this respect, writing a genuinely new book is a challenge. On the one hand, there is a potential overlap with what is well established. On the other, it is difficult to introduce a new subject that needs consolidation in the scientific community. The authors of this book accepted this challenge and, according to the back cover of the book, they had three main objectives: $(a)$ closing the gap between basic condensed-matter science and the forefront of research; $(b)$ bridging complex theoretical concepts and experimental realization; (c) reaching graduate students of various disciplines.

The first goal was certainly achieved, although only on some selected topics of cuttingedge research. Under the umbrella of 'electrons in solids', there is an immense discipline and the forefront is therefore very broad. The five authors selected five topics: (1) electrons on the mesoscopic length scale; (2) interaction of light with electrons (photonics); (3) quantum computing; (4) electron correlation in metal oxides; and (5) topological materials. For all these subjects, the authors give an overview of the new frontier and of the novelties that have appeared in the past few years. However, the five topics are not well balanced in this respect. Quantum computing is obviously the field with the strongest momentum at present, whereas photonics is a more consolidated discipline and the true novelties are not so easily distinguishable.

The second goal of the book is more complicated to ascertain. There is no doubt that the theoretical background is described in close detail and new experimental techniques may be conceived after reading these chapters, but a straightforward connection with the experimental realization is, in general, missing through the book. There are interesting reports of recent experimental results, well connected with the book topics, but this is not a textbook where one would learn how to design and set up new experiments, by discussing the necessities and pitfalls of various techniques. The reader should learn this from other sources.

The third goal is far too ambitious. The textbook is quite complex and too narrowly focused to be adequate for graduate students in areas different from solid-state physics. It would be definitely too demanding for graduate students in engineering, chemistry or material science, and too specialized for students of any other physics subfields (astronomy, high-energy etc.).

If one analyses the book more specifically, a rather heterogeneous style emerges quite clearly. Each author wrote a chapter and, although there is a tentative uniform structure, the level of complexity and, obviously, the writing style differ quite substantially. In 
Chapter 1, focusing on mesoscopic dimensions, the brief introduction is followed by some paragraphs reviewing the basics of electronic structure in the solid state. The complexity of the scope grows at a constant pace and reading Chapter 1 is quite enjoyable. Section 1.2 is a perfect introduction for all the following chapters and it is sensible that it is presented in the first one, although the reader is not necessarily encouraged to read all the chapters or to follow the order of presentation (a typical vice for multi-author/multi-section books). Chapters 2 and 3 also review basic concepts necessary for the understanding of their contents. However, the quantum-mechanical introduction in Chapter 3 is insufficient: naïve on the one hand, and incomplete on the other. In Section 3.1.2, Wikipedia is cited as a source for the history of the abacus. This is inappropriate, given that Wikipedia (as any encyclopaedia) is a tertiary source and secondary sources should be quoted in general unless to show the general perception of a given subject, which was not the case here. In Section 3.1.3, there is a brief review of concepts and keywords of quantum mechanics, but the concept of operator is assumed to be known and the quantum-mechanical postulates are not explicitly stated. This is somewhat disappointing because, as anticipated, the subject of this chapter is certainly the most appealing and it is not in keeping with the third goal of the authors. In Chapter 4, the discussion concerns correlated electrons in transition-metal oxides, which narrows the focus quite substantially. The introduction presents concepts useful also for Chapter 3, although mainly discussed in terms of band theory. I think that the authors should have made more effort to merge the background necessary for Chapters 3 and 4 .

Chapter 4 discusses some well known effects, like superexchange, kinetic exchange, the Jahn-Teller effect and orbital ordering. Incidentally, the Jahn-Teller theorem is not presented in a formally correct way, as one might expect from a textbook for graduate students. In addition, the degree of novelty of the subjects presented here is not so high, given that those effects have been known for a long while, and there is little connection with some recent applications, for example in spintronics. Section 4.8 introduces the role of two techniques, $\mathrm{X}$-ray and neutron scattering, which are quite well established and described in a very rapid way. The necessity of this paragraph is questionable (it is insufficient for a careful reminder of the diffraction techniques and laws, which are too well established to be of inspiration for the development of new experimental techniques). Chapter 5 investigates topological materials. Here there is little introduction to the background which is necessary for the chapter, although one finds many repetitions of band-structure theory. Similarly, Peierls transitions are discussed here as well as in Chapter 4. Section 5.4 is dedicated to topological analysis of electron systems in solids. The definition of topology occurs in a footnote and then it is discussed in Subsection 5.4.1 for the $k$-space. The chapter would have profited from a more general introduction to topology and from mentioning topology in position space, which is the subject of many books and research fields connected with crystallography. Section 5.5 instead is a fascinating survey of all the consequences of electron-electron interactions and it is the best section of this chapter.

In conclusion, I think that this book proposes an interesting overview of the new frontiers in some subfields of solid-state physics, although the topics do not have equal weight and appeal. A defect of the book is some missing homogeneity and an oscillating connection with the modern experimental techniques. The value of the book is the generally high precision of the exposition and clarity of many paragraphs. The stakeholders of this book are likely not graduate students (unless for those in solid-state physics), but post-graduate or even advanced researchers who are looking for an overview of some relatively new fields of research. 